\title{
A RECUPERAÇÃO DE INFORMAÇÃO EM TRABALHOS APRESENTADOS EM ENCONTROS NACIONAIS E REGIONAIS DE ESTUDANTES DE BIBLIOTECONOMIA, DOCUMENTAÇÃO, CIÊNCIA E GESTÃO DA INFORMAÇÃO: UMA PROPOSTA DE UTILIZAÇÃO DO PROGRAMA OPEN CONFERENCE SYSTEMS
}

\author{
Josué Sales Barbosa \\ Lucas Carlos de Oliveira Silva \\ Hugo Oliveira Pinto e Silva
}

\begin{abstract}
Resumo:
O conhecimento científico-tecnológico oriundo de pesquisas visa a solucionar os problemas da sociedade e contribuir para seu desenvolvimento. A partir disso, o processo de comunicação científica, através de canais formais ou informais, torna-se essencial para que a evolução do estado da arte de uma área seja sempre constante. Porém, nem sempre as informações são encontradas nos meios tradicionais de publicação de conhecimento, fazendo com que a literatura cinzenta torne-se uma fonte valiosa para diversas áreas do conhecimento. O desenvolvimento e a evolução das novas tecnologias de informação garantem a essa comunicação um caráter ágil e dinâmico, permitindo que a literatura cinzenta avance para um reconhecimento de sua importância no meio acadêmico. Portanto, faz-se necessário o gerenciamento desse tipo de literatura, que possibilita uma alternativa aos meios tradicionais de aquisição de informação científica. A proposta de criar um ambiente de recuperação de informação e gerenciamento da literatura gerada em encontros de estudantes desenvolve-se a partir da ideia de que, muitas vezes, é possível antecipar tendências que só seriam perceptíveis quando da publicação das mesmas pesquisas em meios já consolidados, muitas vezes ocasionando uma perda de informações existentes apenas em seu andamento, subtraídas quando da redação de artigos ou livros. Assim, a proposta de utilização do programa Open Conference Systems possibilita não somente uma maior agilidade e dinamismo na organização de eventos, tendo também como objetivo a possibilidade para a criação de um ambiente digital em que trabalhos apresentados nos eventos estudantis de biblioteconomia, documentação, ciência e gestão da informação, permitindo uma interação entre pesquisadores, tornando-se uma alternativa para publicação, exercendo função memorial para os eventos estudantis da área e constituir uma fonte de informação relevante para a comunidade.
\end{abstract}

\section{Palavras-Chave:}

Comunicação científica; Tecnologias de informação e comunicação; Literatura cinzenta; Encontros científicos acadêmica

\section{THE INFORMATION RETRIEVAL FROM PAPERS PRESENTED AT NATIONAL AND REGIONAL MEETINGS OF STUDENTS OF LIBRARIANSHIP, INFORMATION SCIENCE AND INFORMATION MANAGEMENT: A PROPOSAL TO USE THE PROGRAM OPEN CONFERENCE SYSTEMS}

\begin{abstract}
:
The scientific-technological knowledge produced by the researches aims at solving problems of the society and contributes to its development. From this on, the process of scientific communication, through formal or informal channels, is essential for the constant evolution of the state of the art of an area. However, not always the information is found in the traditional means of publication, this way, gray literature is a valuable resource for many areas of knowledge. The development and evolution of new information technologies ensure to this kind of communication a dynamic and agile character, allowing the gray literature to reach recognition by the academia. Therefore, it is necessary to manage this type of literature as an alternative to the traditional means of acquiring scientific information. The proposal to create an environment of information retrieval and management of the literature generated
\end{abstract}

(c) Revista Digital de Biblioteconomia e Ciência da Informação,Campinas, v.7, n. 2, p. 59-75, jan./jun. 2010- ISSN: 1678-765X. 
in students' meetings starts from the idea that it is often possible to anticipate trends, that would only be noticed when established means of dissemination publish such researches, often causing a loss of information existing only when the research is in progress or is subtracted when writing articles or books. Thus, the proposed use of the Open Conference Systems program not only allows greater flexibility and dynamism in the organization of events, but also, the development of a digital environment where works presented in the students' events on library, information science and information management could be posted, allowing interaction among researchers, becoming an alternative to publication, assuming a memorial role for students' events in the area, besides being a source of relevant information to the academic community.

\section{Keywords:}

Scientific communication; Information and communication technologies; Gray literature; Scientific and academic meetings 
O conhecimento científico e tecnológico é originado por meio das pesquisas realizadas pelos cientistas, profissionais e estudantes. Estes procuram buscar soluções oriundas dos problemas sociais, políticos ou econômicos, além de contribuir para a diminuição das fronteiras existentes nas diversas áreas do conhecimento.

“Os resultados alcançados por determinado pesquisador são freqüentemente retomados por outros cientistas, teóricos ou aplicados, que dão continuidade ao estudo, fazendo avançar a ciência ou produzindo tecnologias ou produtos neles baseados” (MUELLER, 2000, p.25). Para que essas pesquisas adquiram credibilidade, conforme Mello (1994), os seus resultados devem ser verificados, comunicados, avaliados e utilizados por outros pesquisadores. Isso mostra o caráter cumulativo e parcelar do conhecimento científico e tecnológico.

A comunicação científica e tecnológica corresponde às atividades ligadas à disseminação e uso da informação, desde o início de uma pesquisa até o seu reconhecimento pelos pares. O processo de comunicação ocorre por meio dos canais: formais (publicações com divulgação mais ampla, como periódicos e livros) e informais (comunicações de caráter mais pessoal e a literatura cinzenta).

O avanço das tecnologias da informação, principalmente a partir da década de 80, trouxe inúmeras modificações no fluxo da comunicação científica, em que, até então, predominava a literatura científica impressa, como: mudanças nos canais formais e informais e o surgimento de novas formas de comunicação (correio eletrônico, listas de discussão e videoconferências).

A introdução de novas tecnologias de informação levaram à proposição de um modelo híbrido do processo de comunicação científica, em que a coexistência dos meios impresso e eletrônico constituem fator fundamental. Tal modelo ilustra como as diferentes funções do processo vêm sendo substituídas ou complementadas pelo uso das novas tecnologias. (COSTA, 2000, p.101).

Assim, novas fontes de informações que antes eram desconhecidas ou pouco acessíveis passam a ser amplamente divulgadas por uma comunidade onde se valoriza os contatos mais diretos entre pesquisadores, com trocas de informações valiosas que, muitas vezes, perdem-se quando publicados nos meios tradicionais. É nesse contexto que a literatura 
cinzenta toma fôlego e começa a ser reconhecida como uma importante fonte de informação para o universo acadêmico. Complementamos essa fala com as palavras de Almeida (2000, p. 3), quando esta afirma que “a literatura cinzenta, com seus quase 80 anos de produção e sem uma indexação e divulgação pré-estabelecida, começa a organizar-se com o surgimento e socialização da Internet”, mostrando que o desenvolvimento de novas tecnologias vem auxiliando na divulgação desse tipo de informação.

\subsection{AS TECNOLOGIAS DE INFORMAÇÃO E O MOVIMENTO PARA LIVRE ACESSO À INFORMAÇÃO}

Com o advento das novas tecnologias de informação, todo o universo acadêmico sofreu um grande impacto em toda sua cadeia de produção e divulgação, agilizando processos, otimizando tarefas e fazendo com que resultados que antes dependiam de anos para serem atingidos e divulgados passassem a ser mais rapidamente difundidos. Isso ocorre porque, para Almeida et al (2000, p. 2):

O desenvolvimento da Ciência e Tecnologia reflete a geração de conhecimento bem como a divulgação e sua conseqüente utilização pelos pesquisadores. Tem como ponto de partida o conhecimento científico, que inspira confiabilidade por meio de suas técnicas e métodos utilizados, os quais servem tanto para comunicar os resultados de pesquisa como para se informar acerca dos assuntos em estudo por outros cientistas ou seus pares, garantindo assim a geração da literatura cinzenta.

As características inerentes aos documentos digitais levantam uma série de discussões sobre sua utilização para divulgação de literatura cinzenta, sendo apontadas por diversos autores suas vantagens e desvantagens. Porém, um ponto relevante a ser observado, e que consideramos como primordial, é apontado por Lyman (1997, p. 7) quando afirma que:

The interesting points to watch will be to see if the content of digital and print versions of the same works begin to diverge, and wether readers will be allowed to appropriate published works and reuse them in new contexts. Markets are made by consumers, not by publishers, and the fundamental question concerns the future of reader's behavior as the consumers of information.

Assim, podemos dizer que as tecnologias, ao contribuir para a agilidade da comunicação científica, o reconhecimento e a utilização cada vez maior de literatura cinzenta como fonte de informação em pesquisas, começam a traçar um novo perfil de pesquisador, mais participativo quanto às publicações, muitas vezes buscando canais não somente reconhecidos, mas que possibilitem um diálogo mais próximo com os leitores. Podemos, 
então, dizer que passamos por um período de transição e que os questionamentos que surgem nada mais são do que um reflexo da mudança de comportamento em relação às diferentes gerações de cientistas. Ainda nas palavras de Lyman (1997, p. 9)

The questions concerning technological innovaton might now be reconstituted as a kind of sociology of knowledge: What kind of academic community first created print genres, and was is turn sustained by them? What kind of community is now creating digital genres, and is in turn sistained by them? And what is the relationship between the two, now and in the future?

Portanto, a criação de um ambiente digital para o armazenamento e disseminação dos trabalhos produzidos nos eventos estudantis, onde a cooperação e a troca de ideias aconteça livremente, representa uma forma de democratizar o acesso a informações, criar um memorial dos eventos, aumentar sua visibilidade, tornando-o, futuramente, uma fonte de informação.

Aliado às tecnologias de informação e comunicação, o movimento para acesso livre ao conhecimento potencializa de forma significativa as possibilidades de pesquisa e divulgação de literatura cinzenta, no caso os anais de eventos estudantis. As palavras de Mueller (2006, p. 27) dão a dimensão desse tipo de iniciativa:

\begin{abstract}
O movimento para acesso livre ao conhecimento científico pode ser considerado como o fato mais interessante e talvez importante de nossa época no que se refere à comunicação científica. Ao mesmo tempo, este movimento representa enorme desafio para a comunidade científica, à medida que, quanto mais amplo o seu sucesso, mais radical será a mudança provocada no sistema tradicional e profundamente arraigado de comunicação do conhecimento científico.
\end{abstract}

Porém, é preciso observar que nem sempre essa iniciativa foi vista com bons olhos pela comunidade acadêmica e que só a partir dos anos 1990, com o surgimento dos periódicos de acesso livre e, mais recentemente, após o Budapest Open Access Initiative (BOAI) que aconteceu em 2001 vem se discutindo as diversas questões em torno deste tema. Ainda nas palavras de Mueller (2006, p. 36):

[...] precisamos entender a comunidade científica não só como uma organização social complexa, hierárquica, norteada por princípios e crenças tradicionais, mas também como um contexto em que há vários interesses em jogo. A comunidade se insere em uma sociedade maior, da qual faz parte. As crenças que a norteiam, profundamente estabelecidas, são estimuladas não apenas pela tradição, mas também por interesses de segmentos da própria comunidade e de fora dela, interesses que parecem estar conformando o rumo das publicações eletrônicas. 
Importantes iniciativas e projetos vêm sendo desenvolvidos objetivando oferecer suporte técnico às iniciativas para acesso livre ao conhecimento, como o Public Knowledge Project (PKP), que tem como principais produtos que desenvolve o Open Journal System (OJS) para gerenciamento de periódicos científicos e o Open Conference System (OCS) para eventos e conferências; e a Open Archives Initiative (OAI), que desenvolve padrões de interoperabilidade para otimizar a disseminação de conteúdos.

Já citada anteriormente, a BOAI estabelece em seu texto, de forma breve, quais tipos de textos devem ser disponibilizados para acesso livre e, ainda, suas características no tocante a direitos autorais. Segundo o texto:

The literature that should be freely accessible online is that which scholars give to the world without expectation of payment. Primarily, this category encompasses their peer-reviewed journal articles, but it also includes any unreviewed preprints that they might wish to put online for comment or to alert colleagues to important research findings. There are many degrees and kinds of wider and easier access to this literature. By "open access" to this literature, we mean its free availability on the public internet, permitting any users to read, download, copy, distribute, print, search, or link to the full texts of these articles, crawl them for indexing, pass them as data to software, or use them for any other lawful purpose, without financial, legal, or technical barriers other than those inseparable from gaining access to the internet itself. The only constraint on reproduction and distribution, and the only role for copyright in this domain, should be to give authors control over the integrity of their work and the right to be properly acknowledged and cited. (BOAI, 2002).

Desta forma, a evolução das tecnologias de informação e comunicação, juntamente com o movimento para acesso livre ao conhecimento, potencializa de forma significativa a publicação da literatura cinzenta no ambiente Web. Estes fatores permitem não somente sua disseminação e acesso, mas também a criação de ambientes virtuais de cooperação e colaboração, fazendo com que as barreiras geográficas sejam rompidas e que uma comunidade científica possa estar em constate desenvolvimento.

\section{LITERATURA CINZENTA}

Os canais pelos quais a comunicação científica é divulgada são diversos e podem ser divididos em formais - ou convencionais - e informais. Em um contexto geral do sistema da informação científica e tecnológica, os canais informais possuem relativa importância. Desde a troca de simples e-mails até a formação de comunidades virtuais relacionadas a temas específicos, perpassando por sites pessoais, as possibilidades são cada vez maiores, 
podendo contribuir de maneira efetiva para a difusão do conhecimento científico. Esse tipo de comunicação não apenas promove o acesso às pesquisas, relatórios, estudos e análises, como cria também novas situações que nos meios convencionais não são verificadas.

Assim como os meios convencionais de divulgação são chamados de literatura branca, os meios não convencionais são conhecidos como literatura cinzenta - do inglês Grey Literature. A expressão literatura cinzenta é usada para designar documentos não convencionais. Esses documentos são, em sua maioria, publicados e produzidos nos âmbitos governamental, acadêmico, comercial e da indústria.

Essa forma de comunicação científica não é tão recente. Desde o início do século XX, já era uma recomendação feita do meio científico para o bibliotecário para que fosse incorporada ao acervo da biblioteca.

Diversos autores apontam as características desse tipo de literatura, divergindo em alguns pontos quanto a terminologias, mas podendo ser resumidos com as palavras de Almeida (2000, p. 3), quando a autora diz que:

[...] a literatura cinzenta - documento não convencional, semipublicado, documento escuro, invisível, informal, fugitivo, efêmero, subterrâneo caracteriza-se por ter circulação restrita, assim como acesso e disponibilidade limitados. O referido material não está submetido a um processo de sistematização; apresenta dificuldade de controle bibliográfico e portanto, é de difícil localização, razões pelas quais encontra-se penalizada, economicamente, sua aquisição.

\subsection{DEFINIÇÕES}

As definições para literatura cinzenta variam de acordo com o ano e o ponto de vista do pesquisador. Realizando uma retomada buscando pela origem de sua definição, remontamos ao final da década de 1970, quando, de acordo com Almeida (2000, p. 3):

O termo literatura cinzenta recebe maior significado e torna-se de uso corrente no Seminário de 1978, que se realizou de 13 a 14 de Dezembro, em York (Inglaterra). Sobre tal matéria e segundo afirma Bichiteler, dita expressão foi alcunhada pelos bibliotecários ingleses, popularizando-se na década de 80 .

Seguindo por nossa breve cronologia, no ano de 1983 a Association Française de

(c) Revista Digital de Biblioteconomia e Ciência da Informação,Campinas, v.7, n. 2, p. 59-75, jan./jun. 2010- ISSN: 1678-765X. 
Normalização definiu literatura como:

[...] o documento datilografado ou impresso, com freqüência de caráter provisório dos que se reproduzem e distribuem com um número reduzido de exemplares inferior ao milhar - fora dos circuitos comerciais de distribuição e edição, todo ele a margem dos dispositivos de controle bibliográfico.

Alvarez-Ossorio (1988) citado por Almeida (2000) a define como “... o conjunto de documentos, de tipologia muito variada, que não se publica através dos canais habituais de transmissão científica”. Alberani (1990) considera literatura cinzenta publicações nãoconvencionais, evasivas e, às vezes, efêmeras. Podem incluir, mas não estão limitadas aos seguintes tipos de materiais: relatórios (pré-impresso, preliminar e avançados, técnicos, relatórios estatísticos, memorandos, estudos de mercado, etc), teses, atas de conferências, especificações técnicas e normas, traduções não-comerciais, bibliografias, documentação técnica e comercial, bem como documentos oficiais não publicados comercialmente (principalmente relatórios de governo e documentos).

A Quarta Conferência Internacional sobre Literatura Cinzenta ocorrida em Washington em 1999 a define como: “O que é produzido em todos os níveis do governo, institutos, academias, empresas, indústria, em formato impresso e eletrônico, mas que não é controlado por editores científicos ou comerciais”.

Dessa forma, vemos que a evolução do termo e as discussões sobre suas características são de longa data e que, mesmo com tanto, observamos que só recentemente “a Literatura Cinzenta assume um importante papel na difusão do conhecimento, visto que contém informações jamais encontradas em outro local, além de ser mais disponível (ainda que a uma comunidade restrita) que a literatura convencional” (ALMEIDA; FREITAS, 2004, p. 3).

Isso contribuiu para que os encontros científicos fossem encarados com outros olhos pela comunidade acadêmica, pois os anais produzidos nesses eventos vêm se tornando uma fonte de informação de extrema importância.

\section{ENCONTROS CIENTÍFICOS}

No percorrer de uma pesquisa científica, o pesquisador possui diversas funções, dentre elas está a de comunicador, pois “uma das funções dos cientistas é exatamente a de possibilitar um amplo debate em torno de suas ideias, descobertas, teorias e proposições

(c) Revista Digital de Biblioteconomia e Ciência da Informação,Campinas, v.7, n. 2, p. 59-75, jan./jun. 2010- ISSN: 1678-765X. 
em geral” (CARMO; PRADO, 2005, p.131). Para isso, ele utiliza duas formas de comunicação: a formal e a informal.

A comunicação informal é a mais utilizada no início da pesquisa e caracteriza-se pela presença de contatos pessoais que podem ocorrer de diversas formas: correspondências, contatos por telefone, conversas face a face, encontros científicos, correio eletrônico, listas de discussão, chats e videoconferências.

Os encontros científicos são considerados importantes meios de transferência, avaliação e validação da informação científica e tecnológica, já que reúnem “os membros de uma comunidade científica e/ou técnicos para exporem e discutirem seus trabalhos, envolvendo-os num processo de avaliação que constitui o cerne da atividade de pesquisa.” (CAMPELLO, 2000, p.56).

A organização de um encontro científico e a determinação de seus objetivos, de acordo com Schmidt e Ohira (2002), é feita a partir da análise do contexto econômico, político e social. Ele acaba definindo o tema e os subtemas das atividades que compõem o evento, as quais são: conferências, palestras, comunicações livres, mesas redondas, seminários, vendas de publicações especializadas, exposições de equipamentos, apresentação de filmes científicos e técnicos e painéis.

As principais funções dos encontros científicos, conforme Campello (2000), são: aperfeiçoar os trabalhos apresentados, traçar o estado da arte de uma determinada área do conhecimento e facilitar a comunicação informal entre os participantes.

A apresentação de trabalhos é a principal atividade que ocorre em um encontro científico. Sendo por meio dela que os pesquisadores divulgam os seus estudos, relatos de experiências e trabalhos teóricos, podendo ocorrer de duas maneiras: comunicação oral e em pôsteres.

As principais vantagens da apresentação de trabalhos são: a possibilidade de que sugestões e críticas sejam feitas na hora pelos ouvintes, permissão de uma retroalimentação instantânea, asseguração da prioridade da descoberta científica e facilitação da comunicação pessoal entre os pares.

(c) Revista Digital de Biblioteconomia e Ciência da Informação,Campinas, v.7, n. 2, p. 59-75, jan./jun. 2010- ISSN: 1678-765X. 
As principais desvantagens, conforme Mello (1994), dos encontros científicos são: número excessivo de participantes; programas muito condensados; nível superficial em que são conduzidas as discussões; grande número de trabalhos apresentado, permitindo apenas poucos minutos para apresentação; coincidência das seções paralelas com os horários da apresentação de trabalhos e variação dos padrões de qualidade dos trabalhos apresentados, pois “alguns autores preparam e enviam trabalhos para os encontros, mais em função da oportunidade de viagem do que propriamente por se sentirem impelidos intelectualmente a escrevê-los” (MELLO, 1994. p.36).

Trataremos, agora, do principal veículo de divulgação das apresentações realizadas em eventos, os anais, que têm características e peculiaridades interessantes, sendo levantadas a seguir.

\subsection{OS ANAIS DE ENCONTROS CIENTÍFICOS}

A literatura originada dos encontros científicos é chamada de anais e neles são publicados os trabalhos apresentados e, raramente, as palestras e conferências que ocorreram no encontro.

Os anais são considerados documentos não convencionais que junto com outros (teses, dissertações, relatórios, normas, publicações governamentais) enquadram-se na literatura cinzenta. Esta possui as seguintes características: não é encontrada nos canais comerciais, a informação registrada nela é atualizada, está à margem dos dispositivos de controle bibliográfico, possui difícil localização e é encontrada no formato impresso e eletrônico.

Os anais de eventos possuem algumas características específicas em relação à sua forma de publicação e ao seu conteúdo.

Em relação à publicação, de acordo com Campello (2000) ela pode ser feita pela:

- instituição organizadora, a tiragem dos anais costuma ser pequena e a sua distribuição fica restrita aos inscritos, devido ao elevado custos de produção, o que traz problemas de divulgação e de limitação geográfica. O tratamento e a recuperação dos anais ficam prejudicados, pois a normalização pode ser ineficiente, apresentando falhas nos dados bibliográficos. 
- $\quad$ editoras comerciais: quando são publicados eles são encontrados nos canais normais de venda, não sendo muito freqüente no Brasil.

- $\quad$ publicações periódicas: ocorre quando a entidade organizadora é responsável por uma publicação periódica, sendo que os anais podem ser publicados em um fascículo ou em suplementos.

Em relação ao conteúdo, os anais podem conter textos completos e resumos que, conforme Mello (1994), apesar de omitir informações importantes para as pessoas que não assistiram as apresentações, não podem ser desconsiderados, pois dependendo da área do conhecimento, são a única fonte para a recuperação de alguns trabalhos.

O avanço tecnológico possibilitou o surgimento de novas formas de editoração dos anais, como o OCS, que consiste em um sistema que auxilia na gestão de eventos científicos, facilita a criação de anais e a sua disponibilização no ambiente digital.

\section{Os encontros científicos estudantis}

Os principais encontros científicos da área de Biblioteconomia e Ciência da Informação em que ocorre maior participação de estudantes são os Encontros Regionais dos Estudantes de Biblioteconomia, Documentação, Gestão e Ciência da Informação (EREBDs) e o Encontro Nacional dos Estudantes de Biblioteconomia, Documentação, Gestão e Ciência da Informação (ENEBD).

Esses encontros possuem como objetivo principal: promover a participação, integração e debate dos estudantes de graduação dos cursos de Biblioteconomia, Documentação, Ciência da Informação e Gestão da Informação com estudantes de pós-graduação, docentes, pesquisadores e outros profissionais, visando à difusão do conhecimento, compartilhamento de experiências e intercâmbio social, além de tornarem-se ponto relevante para o aperfeiçoamento da profissão.

Entre os objetivos específicos estão:

- Promover a integração entre os estudantes, estimulando e fortalecendo a comunicação entre estes;

(c) Revista Digital de Biblioteconomia e Ciência da Informação,Campinas, v.7, n. 2, p. 59-75, jan./jun. 2010- ISSN: 1678-765X. 
- Proporcionar o contato dos acadêmicos com temas pouco abordados durante a formação acadêmica;

- Despertar a atenção dos participantes para aspectos não técnicos dos cursos;

- Estimular o pensamento e o raciocínio por meio da oportunidade de discutir temas relacionados à profissão, por meio das apresentações de trabalhos, palestras, conferências, mesas redondas e seminários;

- Aproximar os cursos de Biblioteconomia, Ciência da Informação e Gestão da Informação existentes no país, contribuindo, assim, para o reconhecimento de sua diversidade e particularidades;

- Mostrar o papel do profissional da informação, aprofundando nas suas características, habilidades e oportunidades de trabalho.

As principais vantagens para os estudantes em participar de encontros científicos, conforme Lacerda et al. (2008), são: colaboração na construção do conhecimento, possibilidade de maior convívio no ambiente acadêmico, permissão da troca de ideias e experiências, aumento da segurança, autoestima e valores altruísticos e o reconhecimento do discente na comunidade científica, por meio de apresentação de trabalhos.

\section{O Open Conference System e os eventos estudantis de biblioteconomia: uma proposta de utilização}

Dentro da filosofia do movimento para livre acesso ao conhecimento, propomos a utilização do software OCS para a organização dos eventos estudantis da área de ciência da informação, acreditando que essa ferramenta atenda a todas as expectativas existentes em torno de seu gerenciamento.

Desenvolvido pelo Public Knowledge Project, o OCS é um programa gratuito de publicação Web que cria uma página completa para eventos acadêmicos. Com ele, é possível controlar todo o fluxo de informação de um evento, desde a submissão e avaliação de trabalhos até o arquivamento de apresentações desenvolvidas em diferentes formatos.

Apresentamos abaixo o organograma de funcionamento do programa.

(c) Revista Digital de Biblioteconomia e Ciência da Informação,Campinas, v.7, n. 2, p. 59-75, jan./jun. 2010- ISSN: 1678-765X. 


\section{Conference Managers}

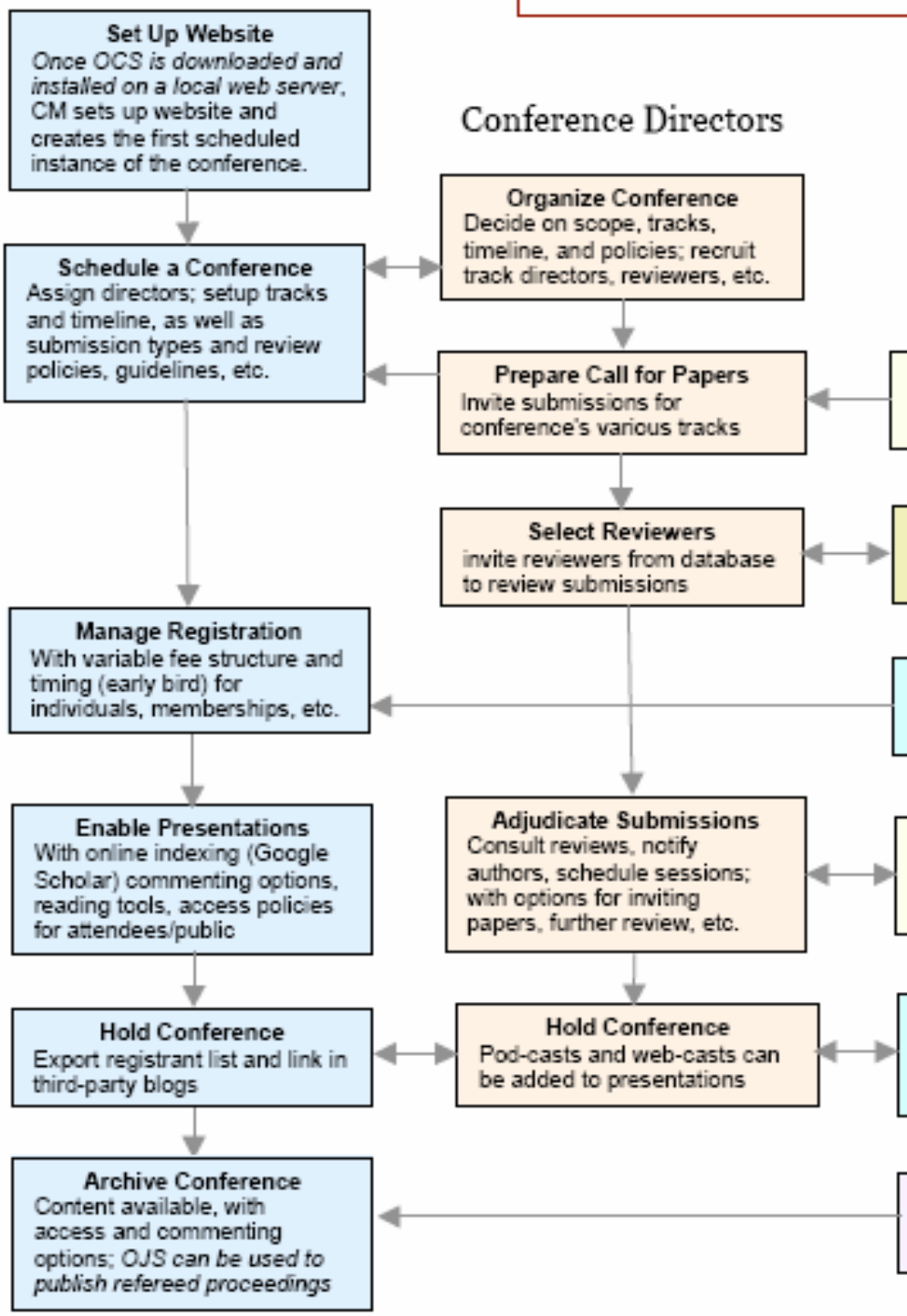

Open Conference Systems 2.0

Open Source Software from the Public Knowledge Project
Authors, Reviewers, Participants

\section{Authors}

Logs into website to upload

abstract and/or fle for review

\section{Reviewers}

one-step log-in for conducting review on the site

Conference Attendees Can use credit card, PayPal, or invoice to cover registration

\section{Authors}

an submit full papers, slides, data sets, etc. after initial acceotance

Attendees and Public Depending on access policies, can comment and blog, in addition to on-site activities

\section{Readers \\ 作} can search and view content

Figura 1: Organograma de funcionamento do Open Conference Systems 2.0 Fonte: Public Knowledge Project

É possível analisar, por meio do organograma, o programa que abrange todos os processos existentes na organização de um evento científico, apresentando ainda o benefício de realizar arquivamento de conferências anteriores e possibilitar um ambiente digital de interação entre os participantes, atendendo aos objetivos deste trabalho que são: criar um ambiente digital de interação com toda a comunidade científica; possibilitar uma alternativa para publicação de trabalhos acadêmicos; além de ser um ambiente de preservação memorial dos eventos, contribuindo para um acompanhamento do estado da 
arte das pesquisas desenvolvidas na graduação pelos discentes e se tornar, com o passar dos anos, uma fonte de informação relevante para a área.

\section{CONSIDERAÇÕES FINAIS}

A utilização das tecnologias de informação vem aumentando em ritmo exponencial desde o início da telemática, expandindo horizontes e permitindo que o conhecimento circule livremente por todo o globo.

A partir desse novo paradigma, o surgimento e a consolidação de novas fontes de informação geram um desconforto por parte dos mais tradicionalistas e ampliam as esperanças dos futuristas e utópicos. Dentro dessa visão, a literatuza cinzenta consolidase como fonte significativa de informação para diversas áreas. Assim, de acordo com Poblacíon, Noronha e Curras (1995, p. 1):

O gerenciamento da literatura cinzenta caracteriza-se como área emergente dentro da ciência da informação. Essa posição vem sendo confirmada por especialistas e profissionais da informação que participam da programação de eventos e aplicam consideráveis recursos para desenvolver estudos sobre a geração e uso desse tipo de literatura.

Dessa forma, garantiremos que a literatura cinzenta seja observada pela área de Ciência da Informação, à luz das novas tecnologias de informação e comunicação, fazendo com que cada vez mais passe a ser observada como uma fonte de informação importante, pois contém informações que não podem ser encontradas em outros meios, expandindo, assim, as possibilidades de acesso e disseminação dentro de nossa sociedade, pois, de acordo com Owen, citado por Almeida (2000, p. 4): 
[...] a sociedade da informação não significa, que a informação se encontra na atualidade dispõe mais facilmente que em outras épocas; ao contrário o que o conceito da "sociedade da informação" parece significar é que necessitamos uma quantidade de informação cada vez maior.

\section{Referências}

ALBERANI, Vilma. et al. The use of grey literature in health sciences: a preliminary survey. Bulletin of the Medical Library Association, v. 78, n. 4, p. 358-363, 1990.

ALMEIDA, Maria do Rosário Guimarães. Comunicação e pesquisa da literatura cinzenta na GreyNet. In: CONGRESSO BRASILEIRO DE BIBLIOTECONOMIA E DOCUMENTAÇÃO, 19., 2000, Porto Alegre. Anais... Porto Alegre : PUCRS, 2000. 20p. Disponível em < http://dici.ibict.br/archive/00000767/01/T115.pdf >. Acesso em: 05 fev. 2010.

ALMEIDA, M. R. G. Literatura cinzenta: teoria e pratica. São Luis: UFMA, 2000. 174p.

ALMEIDA, M. R. G. et al. Gerenciamento da literatura cinzenta para construção de uma base de dados da Universidade Federal do Maranhão - UFMA e Universidade Estadual do Maranhão - UEMA. In: SEMINÁRIO NACIONAL DE BIBLIOTECAS UNIVERSITÁRIAS, 13., 2004, Natal. Disponível em < http://www.cid.unb.br/publico/setores/000/77/materiais/2004/2/72/Ma\%20do\%20Ros\%C 3\%A1rio\%20G\%20Almeida\%20-\%20Gerenc\%20da\%20literatura.pdf > . Acesso em: 05 fev. 2010.

CAMPELLO, B. S. Encontros científicos. In: CAMPELLO, Bernadete Santos; CENDÓN, Beatriz Valadares; KREMER, Jeannette Marguerite. (Orgs.). Fontes de informação para pesquisadores e profissionais. Belo Horizonte: Editora UFMG, 2000. p. $55-71$.

CARMO, J. dos S.; PRADO, P. S. T. do. Apresentação de trabalhos em eventos científicos: comunicação oral e painéis. Interação em Psicologia, Curitiba, v. 09, n.1, 11p. 2005. Disponível em <

http://ojs.c3sl.ufpr.br/ojs2/index.php/psicologia/article/view/3293/2637 >. Acesso em: 05 fev. 2010.

COSTA, S. M. S. Mudanças no processo de comunicação científica: o impacto do uso das novas tecnologias. In: MUELLER, S. P. M.; PASSOS, E. J. L. (Orgs.). Comunicação científica. Brasília: Departamento de Ciência da Informação - UNB, 2000. p. 85 - 104.

LACERDA, A L. de. et al. A importância dos eventos científicos na formação acadêmica dos estudantes de biblioteconomia. Revista ACB: Biblioteconomia em Santa Catarina , Florianópolis, v.13, n.1, p.130-144, 2008. Disponível em < http://revista.acbsc.org.br/index.php/racb/article/view/553/678 >. Acesso em: 5 fev. 2010.

LYMAN, Peter. Digital documents and the future of the academic community. In: PROCEEDINGS FROM THE CONFERENCE ON SCHOLARLY COMMUNICATION AND TECHNOLOGY, 1997, Atlanta. Anais... Disponivel em < 
http://www.eric.ed.gov/ERICDocs/data/ericdocs2sql/content_storage_01/0000019b/80/15 /1c/78.pdf >. Acesso em: 05 fev. 2010.

MELLO, L. L. C. C. de. Anais de encontros científicos com fonte de informação. 1994. 132 f. Dissertação (Mestrado em Ciência da Informação) - Escola de Biblioteconomia da UFMG, Universidade Federal de Minas Gerais, Belo Horizonte, 1994.

MUELLER, S. P. M. A ciência, o sistema de comunicação científica e a literatura científica. In: CAMPELLO, Bernadete Santos; CENDÓN, Beatriz Valadares; KREMER, Jeanette Marguerite. (Orgs.). Fontes de informação para pesquisadores e profissionais. Belo Horizonte: Editora UFMG, 2000. p. 21-34.

MUELLER, S. P. M. A comunicação científica e o movimento de acesso livre ao conhecimento. Ciência da Informação, Brasília, v. 35, n. 2, p. 27-38, 2006. Disponível em $<$ http://revista.ibict.br/ciinf/index.php/ciinf/article/view/826/668 $>$. Acesso em: 5 fev. 2010.

OHIRA, M. L. B.; SCHMIDT, L. Bibliotecas virtuais e digitais: análise das comunicações em eventos científicos (1995/2000). Revista ACB: Biblioteconomia em Santa Catarina, Florianópolis, v. 7, n.1, p. 73-97, 2002. Disponível em < http://revista.acbsc.org.br/index.php/racb/article/view/377/456 >. Acesso em: 5 fev. 2010. OPEN Archives Initiative. Disponível em: <http://www.openarchives.org/>. Acesso em: 04 abr. 2009.

POBLACION D. A.; NORONHA, D. P. CURRAS, E. Literatura cinzenta versus literatura branca. Ciência da Informação, Brasília, v. 25, n. 2, p.1-10, 1995. Disponível em $<$ http://revista.ibict.br/ciinf/index.php/ciinf/article/view/442/400 $>$. Acesso em: 5 fev. 2010.

PUBLIC Knowledge Project. Disponível em: <http://pkp.sfu.ca/>. Acesso em: 04 abr. 2009.

THE BUDAPEST OPEN ACCESS INITIATIVE (Budapeste). Declaração de Budapeste. 14 fev 2002. Disponível em: < http://www.soros.org/openaccess/read.shtm I>. Acesso em: 04 abr. 2009. 


\section{Josué Sales Barbosa}

Discente de Biblioteconomia da Universidade Federal de Minas Gerais - UFMG

josuesbarbosa@gmail.com

\section{Lucas Carlos de Oliveira Silva}

Discente de Biblioteconomia da Universidade Estadual Paulista - UNESP

otimeia@marilia.unesp.br

\section{Hugo Oliveira Pinto e Silva}

Discente de Biblioteconomia da Universidade Federal de Minas Gerais - UFMG hugoopes@yahoo.com.br

Recebido em: 14/05/2009

Aceito para publicação em: jul/2009 